\title{
A study of neovascularization in the rat ischemic hindlimb using Araldite casting and Spalteholtz tissue clearing
}

\author{
Peter E. Westerweel ${ }^{\mathrm{a}, 1}$, Maarten B. Rookmaaker ${ }^{\mathrm{a}}$, Anton-Jan van Zonneveld ${ }^{\mathrm{b}}$, \\ Ronald L.A.W. Bleys ${ }^{\mathrm{c}}$, Ton J. Rabelink ${ }^{\mathrm{b}}$, Marianne C. Verhaar ${ }^{\mathrm{a}, *, 2}$ \\ ${ }^{a}$ Department of Vascular Medicine, University Medical Center Utrecht, 3584 CX Utrecht, The Netherlands \\ ${ }^{\mathrm{b}}$ Department of Nephrology and Hypertension, Leiden University Medical Center, Leiden, The Netherlands \\ ${ }^{c}$ Department of Pharmacology and Anatomy, University Medical Center Utrecht, Utrecht, The Netherlands
}

Received 1 September 2004; received in revised form 11 July 2005; accepted 19 July 2005

\begin{abstract}
Introduction: Understanding neovascularization is an important prerequisite for therapeutic advances aimed at the salvation of ischemic tissues. We explored an alternative strategy to corrosion casting for visualizing neovascularization in a rat hindlimb ischemia model. Methods: Rats were subjected to hindlimb ischemia by femoral artery ligation. Directly after femoral artery occlusion and at 7 , 14, 21 and 28 days postsurgery, rats were sacrificed, and neovascularization was evaluated by vascular casting with Araldite plastic and subsequent Spalteholtz tissue clearing. Results: Semitransparent preparations were obtained, in which the casted arteries could be directly and three-dimensionally visualized in detail and in relation to the surrounding tissue. In the vascular casts, collateral formation and recanalization of previously thrombosed arteries were demonstrated. Conclusions: We describe an alternative approach to study neovascularization in animal models. This method, which combines Araldite plastic vascular casting with Spalteholtz tissue clearing, preserves all vasculature as well as the surrounding tissue. In a small time series in rat ischemic hindlimbs, we show that restoration of blood flow after ischemia not only involves newly formed collaterals, but also recanalization of previously thrombosed arterial segments. (C) 2005 Elsevier Inc. All rights reserved.
\end{abstract}

Keywords: Investigative techniques; Models, Animal; Neovascularization, Physiologic; Arterial occlusive disease

\section{Introduction}

Neovascularization is a physiological response to ischemic injury, which alters both the microvasculature in and the conduit arteries leading into the jeopardized tissue. Despite this response, arterial occlusive disease with subsequent tissue ischemia and necrosis remains a prominent clinical problem. Enhancing neovascularization

\footnotetext{
* Corresponding author. Tel.: +31 30 2509815; fax: +31 302518328 .

E-mail address: m.c.verhaar@azu.nl (M.C. Verhaar).

${ }^{1}$ Peter E. Westerweel is a research fellow of the Dr. E. Dekker program (2004T022) of the Dutch Heart Foundation.

${ }^{2}$ Marianne C. Verhaar is supported by the Netherlands Organization for Scientific Research (NWO VENI-grant 016.036.041).
}

is a promising new strategy for treating ischemic disease $[1,2]$. Visualizing neovascularization is important to study this process. Corrosion casting may be used; however, this technique renders vulnerable preparations, and the surrounding tissue is lost in the procedure. We employed an alternative approach for visualizing vasculature, by vascular casting of the blood vessel system with Araldite plastic in combination with Spalteholtz tissue clearing, a chemical procedure to preserve the surrounding tissue and make it transparent [3]. This allows detailed observation of the vascular morphology in its original three-dimensional context. We used this method to further characterize the process of neovascularization in rats with hindlimb ischemia induced by femoral artery ligation, a commonly used animal model for studying human peripheral vascular disease [4]. 


\section{Methods}

\subsection{Animal model}

Six male Wistar rats weighing 350-450 g (Harlan, the Netherlands) were used for the experiments. The study protocol was approved by the animal ethics committee of the University of Utrecht, and all experiments were performed in accordance with accepted institutional and governmental policies. Rats were anaesthetized using $\mathrm{N}_{2} \mathrm{O}$ /isoflurane gas. A skin incision was made on the medial side of the thigh of the left hindlimb, and the femoral artery was dissected free without damaging the vein and nerve. The left femoral artery was ligated with two sutures using 3-0 woven silk, just distal to the inguinal ligament. The wound was closed, and the animals were allowed to recover. The animals were closely monitored by veterinary staff and received buprenorphin analgesic medication $(0.05 \mathrm{mg} / \mathrm{kg}$ at 8 -h intervals $)$ for 2 days following surgery. The rats were sacrificed for Araldite casting immediately after surgical induction of hindlimb ischemia and at Day 7, 14, 21 and 28 postsurgery.
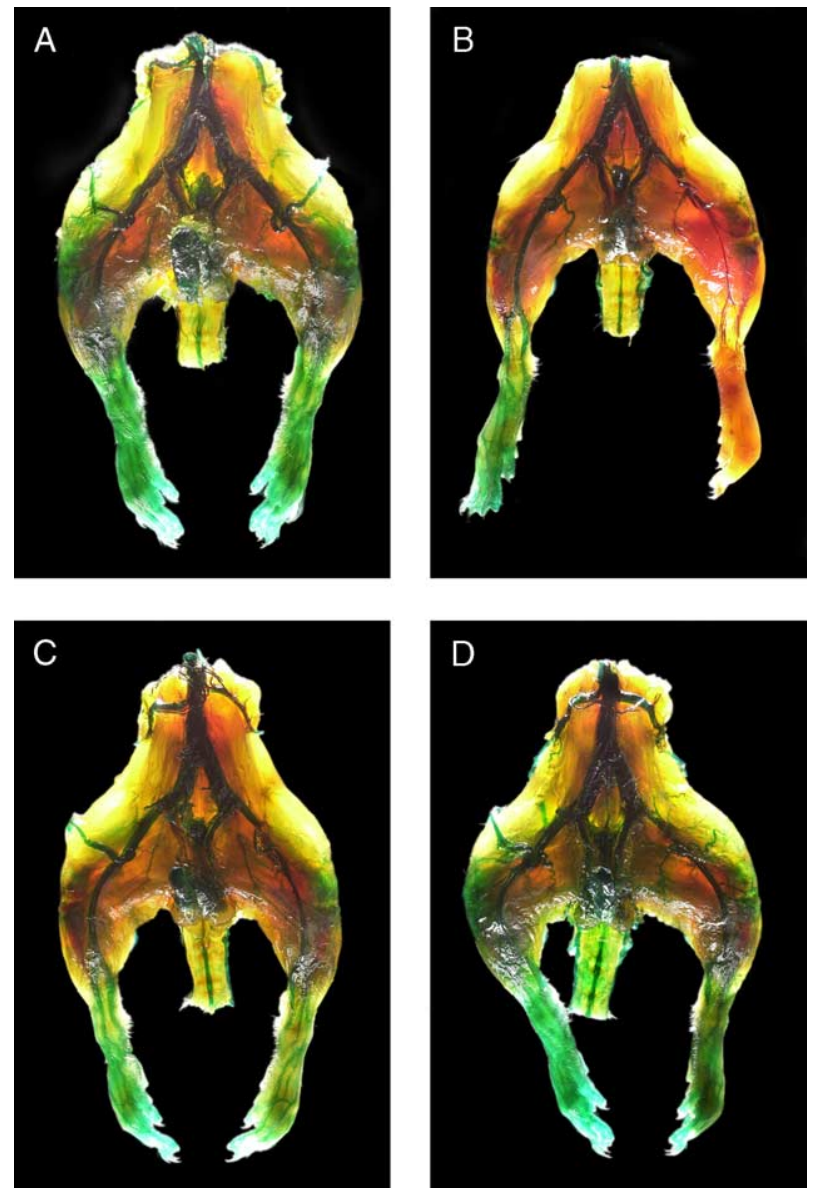

Fig. 1. (A-D) Series of Araldite preparations photographed from the ventral side, made in a control rat $(\mathrm{A})$ and after induction of ischemia by ligation of the left femoral artery. Seven days after ligation (B), there is profound ischemia on the ligated side, and distal arteries are thrombosed. After 14 days (C), collaterals have formed and flow is restored in the original conduit arteries. Total recovery has taken place after 21 days (D).
One rat served as additional control with no hindlimb ischemia on either side.

\subsection{Araldite casting}

At different time points after the induction of hindlimb ischemia, rats were anaesthetized with pentobarbital (60 $\mathrm{mg} / \mathrm{kg}$ ), and the abdominal cavity was opened. The aorta was cannulated between the renal arteries and the aortic bifurcation, the animals were bled and the vascular system was flushed using prewarmed (temperature, $37^{\circ} \mathrm{C}$ ) physiological saline containing $0.2 \mathrm{mg}$ of nitroglycerine to dilate the blood vessels and remove residual blood. A mixture of green-coloured Araldite F, Hardener HY 2967 and dilutioner DY 026 SP (Ciba Geigy, Basel, Switzerland) was prepared according to the manufacturer's instructions and infused through the same cannula at a constant pressure of $100 \mathrm{~mm} \mathrm{Hg}$ until the onset of polymerization. The onset of polymerization was determined by observation of the solidification of the Araldite in the infusion bottle. The temperature of the Araldite mixture was kept constant at approximately $20^{\circ} \mathrm{C}$. During infusion, venous return of Araldite was observed in all veins, consistent with the known characteristic of Araldite to fully penetrate the capillary bed and resulting in filling of both arteries and veins with Araldite.

\subsection{Spalteholtz tissue clearing}

Skin and subcutaneous fat were carefully removed for better visualization. The hind portion of the rat was placed in a series of alcohol solutions of increasing concentration (70\%, 80\%, 96\% and $100 \%$, respectively) to dehydrate the surrounding tissue. The solutions were refreshed daily, and a higher concentration was used each fourth day. The preparations were finally placed in pure methyl benzoate.

\section{Results}

We obtained preparations in which the vascular system could be studied in detail at all levels in the tissue. Figs. 1 and 2 demonstrate Araldite castings in semitransparent models of rats at the following time points: prior to ligation and at 7, 14 and 21 days after acute femoral artery occlusion. Particularly when transluminated as in the figures, the green-coloured Araldite contrasts clearly with the surrounding tissue and can be visually discerned from filling of the blood vessels by thrombi.

In the control rat, the normal distribution of arterial supply to the hindlimb could be observed. As in humans, the common iliac artery bifurcates in the internal iliac artery supplying the inguinal and pelvic region, and the external iliac artery, with a slightly larger caliber, that mainly forms the femoral artery and predominantly vascularizes the hindlimb (Fig. 1A). In the casting obtained immediately 


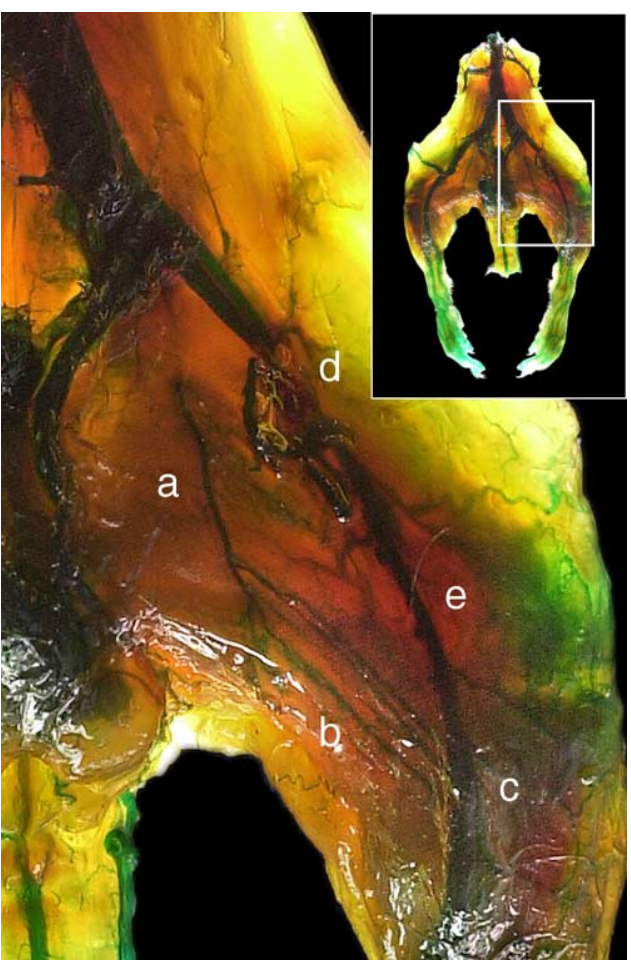

Fig. 2. Detail of the Araldite casting made after 14 days (2.5-fold magnification from Fig. 1C) in which a collateral artery originating from the inguinal region is shown in detail (a), with several collaterals branching of (b) and connecting with the femoral artery (c) distal to the place of ligation (d). The originally thrombosed femoral artery is refilled with Araldite (e)

after ligation, no Araldite was present distal to the site of occlusion, indicating hypoperfusion of the ischemic limb. A thrombus could be observed in the femoral artery and vein distal to the ligation (not shown). After 7 days of occlusion, there was a minimal increase of Araldite content of the ischemic limb consistent with a minimal increase in perfusion. A thrombus was still present in the femoral vessels (Fig. 1B). At 14 days after femoral artery occlusion, the Araldite penetration in the previously ischemic limb had substantially improved. In the area surrounding the ligation, there was a marked increase in the number and caliber of blood vessels. Some of these vessels had the typical 'corkscrew' appearance of newly formed collateral arteries. The caliber of the left internal iliac artery had increased compared to the contralateral artery and the nonligated control. The inguinal region of the ischemic limb also showed an increased number of visible arteries of variable size. Several of these connected to the distal part of the femoral artery. At this time point, the section of the femoral artery distal to the ligation no longer contained a thrombus, but was completely filled with Araldite (Fig. 1C). At 21 days (Fig. 1D) and 28 days (not shown) after occlusion, the Araldite penetration of both limbs was equal and comparable to the control rat. Interconnecting arteries originating from the inguinal vasculature were seen in all three preparations at 14, 21 and 28 days after ligation (Figs. 1C-D and 2). In the preparations containing interconnecting collaterals, the previously thrombosed distal section of the femoral artery was filled with Araldite. No thrombus remained in either the original femoral artery or its distal branches (Fig. 1C-D).

For proper preparation, skin and subcutaneous fat were removed. In these tissues, Araldite-filled vessels could also be observed. Acutely after surgical induction of ischemia, there was a marked reduction in Araldite content of the dermis and subcutaneous fat, similar to the hypoperfusion observed in the deeper vessels. In the preparation made on Day 7 following ligation, the superficial vasculature appeared to be more extensively penetrated by the Araldite than the deeper vasculature (not shown). In later preparations, this gradually increased in conjunction with the increase in perfusion in the deep vasculature.

\section{Discussion}

We report that vascular casting with Araldite plastic with Spalteholtz tissue clearing is an effective method to visualize neovascularization in a rat model of hindlimb ischemia with preservation of the surrounding tissues.

Araldite plastic has several important physical characteristics that make it suitable for studying the anatomy of small-sized vasculature. Araldite has a dynamic viscosity of 1.5 times the blood viscosity and a polymerization time of $45 \mathrm{~min}$, which allows adequate filling of approximately $90 \%$ of the arterioles with a diameter of $15 \mu \mathrm{m}$. It thus penetrates deeply in the tissue, which is illustrated by the occurrence of venous return. Araldite-perfused tissue is suitable for scanning electron microscopy investigation and histological morphometrical examination [5]. Spalteholtz tissue clearing is a classical technique for producing transparent three-dimensional tissue specimens which was introduced by Spalteholtz [3] almost a century ago. After complete dehydration, the tissue is impregnated with methylbenzoate. Because methylbenzoate has a refractory index that approximates that of the tissue, this procedure will provide transparent three-dimensional preparations [6].

Using our method in a rat model of acute femoral artery occlusion, we observed neovascularization and subsequent recovery of perfusion via collaterals, consistent with previous reports $[7,8]$. Interestingly, in the preparations made early after induction of ischemia (directly postoperatively and at Day 7), a thrombus was present in the part of the ligated femoral artery distal to the site of occlusion. At later time points, Araldite filling of the femoral artery distal to the surgically induced ligation could be observed. This suggests that thrombotic segments underwent recanalization and resumed their conduit functions. This process appears to occur in conjunction with the formation of interconnecting collaterals, since collaterals connecting the proximal segment to the distal segment of the occluded vessel were present at later time points. Recanalization following arterial occlusion has been reported to occur in humans. In patients 
with a cerebral occlusion, spontaneous recanalization was observed in approximately $17 \%$ of all individuals [9]. In a recent retrospective review of 451 patients who underwent repetitive arteriography, the incidence of this phenomenon was less than $2 \%$ in occlusions of arteries in the lower extremity [10]. It is remarkable that in our small series, we consistently observed this process.

Several techniques may be used to study vascular morphology. Angiography is frequently used for assessment of arteries or veins, and offers quantitative results. However, it provides a two-dimensional representation of the vasculature, with potential overprojection and misinterpretations of the anatomy. Furthermore, standard radiographic images have a limited resolution and are not suitable for visualizing medium- and smaller-sized vessels in rats. Recent advances in technology have increased the resolution, but require highly specialized equipment [11]. Corrosion casting allows visualization of small arteries $<100 \mu \mathrm{m}$ in diameter [8,12], but the casts are vulnerable and many of the smaller vessels are lost in the procedure. Araldite casting combined with tissue clearing has important advantages over corrosion casting or angiography. Small- and mid-sized vessels are preserved and can be adequately visualized. Many of the collaterals that developed in response to ischemia in our study were indeed small or mid sized. In addition, because the surrounding tissue is not removed, the anatomical orientation remains intact and the soft tissues can be observed. In our study, we noted the occurrence and resolution of a thrombus in the occluded arterial segment. Both angiography and corrosion casting do not provide information on the nature of the filling of nonperfused vasculature.

Our study has several limitations. Although the method we present provides important information on the vascular anatomy in relation to the surrounding tissue, it provides only qualitative data. It is possible to quantify the vessel density of our preparations by placing a grid placed over a photograph of the preparation and counting intersections, as is commonly done for calculating an angiographic score. However, for quantification of the recovery after induction of hindlimb ischemia, other techniques may be more suitable, e.g., using microsphere perfusion, laser Doppler flow measurements and high-resolution angiography $[7,8,13,14]$. Another limitation is that the obtained threedimensional preparations are best appreciated when directly analyzed. Finally, we did not establish the reproducibility of the measurements in this study.

In summary, we have demonstrated an alternative technique to corrosion casting for direct visualization of (neo)vascularization. By combining Araldite plastic vascular casting with Spalteholtz tissue clearing technique of the soft tissues, semitransparent preparations can be obtained. Using this approach in the rat model of acute hindlimb ischemia, we observed collateral formation as well as the consistent occurrence of recanalization.

\section{Summary}

To study neovascularization in a rat model of hindlimb ischemia, we combined Araldite vascular casting and Spalteholtz tissue clearing, creating semitransparent preparations that allow direct detailed visualization of arteries. Using this technique in a time series of hindlimb ischemia, we demonstrate collateral formation, collateral arteries interconnecting to occluded arteries distal to the experimental ligation and recanalization of previously thrombosed arteries.

\section{Acknowledgments}

We thank B. van Middelaar for excellent technical assistance with the animal experiments.

\section{References}

[1] Losordo DW, Dimmeler S. Therapeutic angiogenesis and vasculogenesis for ischemic disease: Part II Cell-based therapies. Circulation 2004; 109:2692-7.

[2] Losordo DW, Dimmeler S. Therapeutic angiogenesis and vasculogenesis for ischemic disease: Part I Angiogenic cytokines. Circulation 2004; 109:2487-91.

[3] Spalteholtz W. Ueber das Durchsichtigmachen von menschlichen und tierischen praeparaten. Leipzig: Hirzel, 1914.

[4] Couffinhal T, Silver M, Zheng LP, Kearney M, Witzenbichler B, Isner JM. Mouse model of angiogenesis. Am J Pathol 1998;152:1667-79.

[5] van der Zwan A, Hillen B. Araldite F as injection material for quantitative morphology of cerebral vascularization. Anat Rec 1990;228: 230-6.

[6] Steinman WF. Makroskopische Prapartionsmethoden in der Medizin. Stuttgart: Georg Thieme Verlag, 1982.

[7] Hershey JC, Baskin EP, Glass JD, Hartman HA, Gilberto DB, Rogers IT, Cook JJ. Revascularization in the rabbit hindlimb: dissociation between capillary sprouting and arteriogenesis. Cardiovasc Res 2001; 49:618-25.

[8] Herzog S, Sager H, Khmelevski E, Deylig A, Ito WD. Collateral arteries grow from preexisting anastomoses in the rat hindlimb. Am J Physiol Heart Circ Physiol 2002;283:H2012-20.

[9] Kassem-Moussa H, Graffagnino C. Nonocclusion and spontaneous recanalization rates in acute ischemic stroke: a review of cerebral angiography studies. Arch Neurol 2002;59:1870-3.

[10] Gargiulo NJ, Veith FJ, Lipsitz EC, Ohki T, Suggs WD, Cayne NS, Dadian N, Wain RA. Spontaneous recanalization of arterial occlusions: an unusual mechanism for symptomatic improvement. J Vasc Surg 2002;36:1161-6.

[11] Takeshita S, Isshiki T, Mori H, Tanaka E, Eto K, Miyazawa Y, Tanaka A, Shinozaki Y, Hyodo K, Ando M, Kubota M, Tanioka K, Umetani K, Ochiai M, Sato T, Miyashita H. Use of synchrotron radiation microangiography to assess development of small collateral arteries in a rat model of hindlimb ischemia. Circulation 1997;95:805-8.

[12] Conrad MC, Anderson JL, Garrett JB. Chronic collateral growth after femoral artery occlusion in the dog. J Appl Physiol 1971;31:550-5.

[13] Couffinhal T, Silver M, Zheng LP, Kearney M, Witzenbichler B, Isner JM. Mouse model of angiogenesis. Am J Pathol 1998;152:1667-79.

[14] Yang HT, Deschenes MR, Ogilvie RW, Terjung RL. Basic fibroblast growth factor increases collateral blood flow in rats with femoral arterial ligation. Circ Res 1996;79:62-9. 